\title{
The Association between Earlobe Creases and Cardiovascular Events in Japanese Hemodialysis Patients: A Prospective Cohort Study
}

\author{
Minako Wakasugi ${ }^{1}$, Masaaki Nagai ${ }^{2}$, Saori Yokota $^{3}$, Kentaro Omori ${ }^{3}$, Hirokazu Fujikawa ${ }^{4}$, \\ Ikuo Aoike ${ }^{5}$, Tsukasa Omori ${ }^{3}$, Junichiro James Kazama ${ }^{6}$ and Ichiei Narita ${ }^{7}$
}

\begin{abstract}
:
Objective The earlobe crease, a wrinkle extending from the tragus to the outer border of the earlobe, is a well-known surrogate marker for a high risk of cardiovascular disease. However, information is lacking about its association with cardiovascular events among hemodialysis patients, who already have an increased risk of cardiovascular disease. We tested the hypothesis that earlobe creases are independently associated with the risk of cardiovascular events among Japanese hemodialysis patients.

Methods This prospective cohort study followed 247 adult hemodialysis patients with no history of cardiovascular disease for 4 years. The presence of earlobe creases was defined by two researchers using photos of patients' earlobes on both sides while blinded to one another's assessments and clinical data. The primary outcome was defined as the first fatal or nonfatal cardiovascular event (myocardial infarction, ischemic or hemorrhagic stroke, or peripheral vascular disease requiring aortic or peripheral vascular bypass surgery or below- or above-the-knee amputation). A Fine-Gray competing risks regression model was used to examine the association between earlobe creases and cardiovascular events.

Results During the 4-year follow-up period, 43 patients suffered cardiovascular events. After the competing risk of non-cardiovascular death was accounted for, patients with earlobe creases had an increased cumulative incidence of cardiovascular events compared to those without earlobe creases (subhazard ratio $=2.04,95 \%$ confidence interval: 1.09 to 3.82). This association was no longer significant after adjusting for age.

Conclusion Earlobe creases were not independently associated with cardiovascular events among Japanese hemodialysis patients, suggesting that these marks are simply indicative of advanced age.
\end{abstract}

Key words: cardiovascular disease, earlobe crease, hemodialysis patients, survival analysis

(Intern Med 59: 927-932, 2020)

(DOI: 10.2169/internalmedicine.3943-19)

\section{Introduction}

Dialysis patients have a much higher risk of cardiovascular mortality than the general population, with a 7 to 30 times higher age-adjusted cardiovascular mortality than that within the general population $(1,2)$. Despite the recent de- cline in cardiovascular mortality in dialysis patients (3-5), cardiovascular disease (CVD) is still one of the leading causes of death among dialysis patients in many countries, including Japan (6). A simple, reliable screening marker to identify individuals with a high risk of CVD is therefore necessary for use in clinical settings.

The earlobe crease (ELC), also called Frank's sign (7), is

${ }^{1}$ Division of Comprehensive Geriatrics in Community, Niigata University Graduate School of Medical and Dental Sciences, Japan, ${ }^{2}$ Department of Internal Medicine, Sakura Clinic, Japan, ${ }^{3}$ Department of Internal Medicine, Medical Corporation Group Omori Medical Clinic, Japan, ${ }^{4}$ Department of Internal Medicine, Maihira Clinic, Japan, ${ }^{5}$ Department of Internal Medicine, Koyo Medical Clinic, Japan, ${ }^{6}$ Department of Nephrology and Hypertension, Fukushima Medical University, Japan and ${ }^{7}$ Division of Clinical Nephrology and Rheumatology, Niigata University Graduate School of Medical and Dental Sciences, Japan

Received: September 19, 2019; Accepted: October 29, 2019; Advance Publication by J-STAGE: December 13, 2019

Correspondence to Dr. Minako Wakasugi, minakowa@med.niigata-u.ac.jp 
a wrinkle extending from the tragus to the outer border of the earlobe. Several studies in non-dialysis populations have described ELC as a predictor of future CVD, including coronary heart disease (8-11), cerebrovascular disease (12), and peripheral vascular diseases $(13,14)$. The presence of ELC, which can be assessed easily without any test, might function as a visible prompt for both physicians and patients to screen for CVD. We previously reported a cross-sectional study showing that the prevalence of ELC was not associated with prevalent CVD in Japanese hemodialysis patients (15). However, survivor bias may have affected the results of that study.

To our knowledge, no previous studies have evaluated the association between ELC and cardiovascular events among hemodialysis patients. Accordingly, this prospective cohort study assessed the hypothesis that ELC is associated with the risk of cardiovascular events among Japanese hemodialysis patients, independent of chronological age.

\section{Materials and Methods}

\section{Study design and participants}

This study was conducted as a follow-up to a previously reported cross-sectional study (15); a detailed description of this cohort has been previously published. In brief, individuals undergoing hemodialysis were recruited from five outpatient hemodialysis centers in Niigata, Japan, between February and November 2012. Eligibility criteria for participating patients were as follows: 1) $\geq 20$ years old, 2) receiving maintenance hemodialysis 3 times a week and capable of understanding and giving written informed consent to participate in the study, and 3) no history of CVD at baseline. Potential participants were excluded if they had ear piercings.

All participants provided their written informed consent to participate in the study. This study was conducted in compliance with the Declaration of Helsinki and was approved by the Institutional Review Board Ethics Committee of the Niigata University Graduate School of Medicine.

\section{Predictors}

The predictor variable was the presence of ELC at baseline, as defined according to previously reported methods (15). In brief, bilateral earlobe photographs were taken by one researcher with the patient in a supine position during a dialysis session. Each digital photo was assessed independently by two researchers who were blinded to one another's assessments and to the clinical data in order to determine the presence of ELC. The Kappa coefficients for interrater agreement were moderate for ELC presence $[\kappa=0.67$, 95\% confidence interval (CI): $0.56-0.78]$, and discrepancies were resolved through consensus with a third researcher.

\section{Covariates and outcome data}

Data on baseline patient characteristics, including cause of end-stage kidney disease (ESKD), were collected by reviewing medical records. Smoking was defined as the current daily use of cigarettes. Dialysis vintage was calculated as the time between the very first dialysis session and the day that the earlobe photographs were taken. Laboratory data, which were measured at the first dialysis session of each week, were obtained from patient medical records. The nonhigh-density lipoprotein (HDL) cholesterol concentration was calculated as the difference between the total cholesterol concentration and the HDL cholesterol concentration.

The primary outcome measure of this study was the occurrence of the first fatal or nonfatal cardiovascular event during follow-up. Cardiovascular events included myocardial infarction, ischemic or hemorrhagic stroke, and peripheral vascular disease that required aortic or peripheral vascular bypass surgery or below- or above-the-knee amputation. Repeat cardiovascular events were not included. The secondary outcome measure was all-cause mortality. Information about cardiovascular events and death was obtained by reviewing patient medical charts.

\section{Statistical analyses}

Power analyses were performed using the PASS software program (Jerry Hintze, Kayville, USA). A two-sided logrank test was used to account for competing risks, with an overall sample size of 243 patients (165 patients without ELC and 78 patients with ELC) calculated to achieve $90 \%$ power at a 0.05 significance level for the detection of a hazard ratio of 2.0. The hazard ratio was set to 2.0, as too small an effect size would have no meaning in clinical settings.

The baseline characteristics of patients with or without ELC were expressed as the mean [standard deviation (SD)] or median (25th and 75th percentiles) for continuous variables and as percentages for categorical variables. Differences in clinical characteristics with and without ELC were compared using the chi-square test or Fisher's exact test for categorical data and Student's $t$-test or the Mann-Whitney $U$-test for continuous variables. Pearson's and Spearman's correlation coefficients were calculated to evaluate relationships between variables.

Because hemodialysis patients also have high noncardiovascular mortality rates $(1,5,16)$, we used the FineGray model of competing risk regression analyses to compare event rates of cardiovascular disease among patients with and without ELC. We first adjusted for the age before adding the patient sex into the model. Finally, we adjusted for the propensity score (PS) as a linear covariate due to the limited size of the study cohort (17). The PS was calculated using a logistic regression analysis to consider each individual's probability of exposure to confounding variables, including age, sex, dialysis vintage (log-transformed), cause of ESKD, smoking status, and pre-dialysis serum hemoglobin, albumin, non-HDL cholesterol, and C-reactive protein concentrations (log-transformed). In sensitivity analyses, deaths due to unknown causes were classified as CVD deaths.

The Kaplan-Meier method and restricted mean survival 


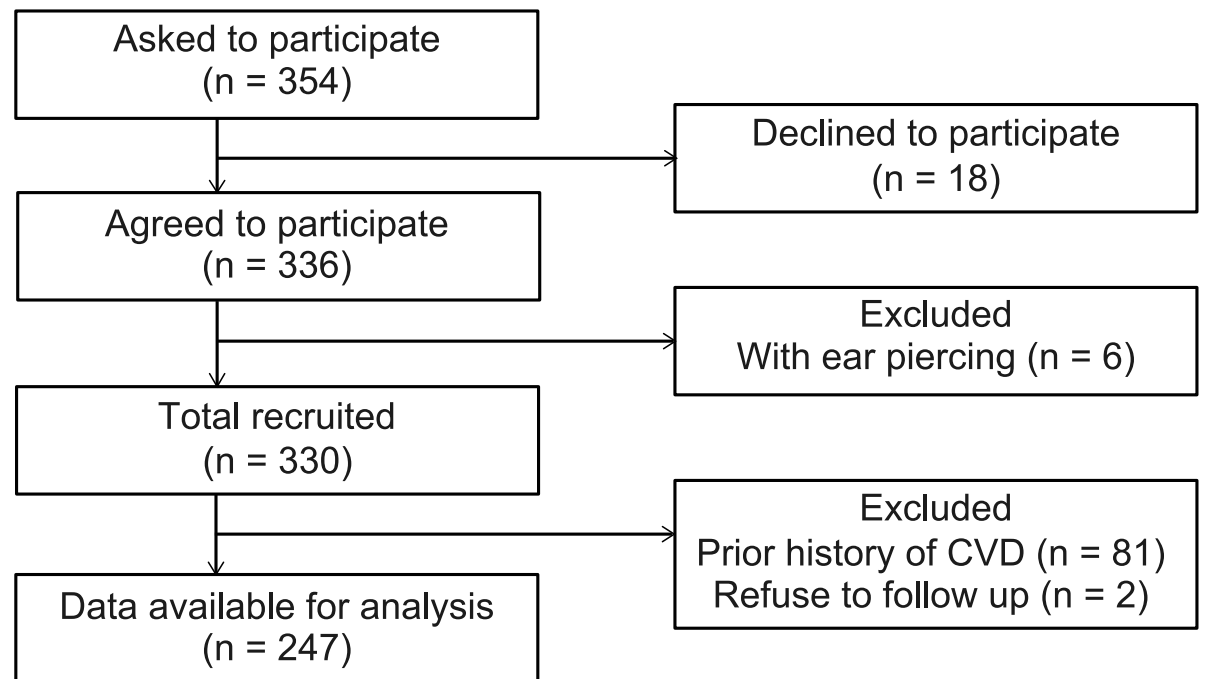

Figure 1. Flowchart of study participant selection.

Table 1. Baseline Characteristics of Hemodialysis Patients with No History of Cardiovascular Disease Stratified by the Presence or Absence of Earlobe Creases.

\begin{tabular}{|c|c|c|c|c|}
\hline \multirow[b]{2}{*}{ Baseline characteristics } & \multirow{2}{*}{$\begin{array}{l}\text { All patients } \\
\quad(\mathrm{n}=247)\end{array}$} & \multicolumn{2}{|c|}{ Earlobe creases } & \multirow[b]{2}{*}{$\mathrm{p}$ value } \\
\hline & & $\begin{array}{c}\text { Present } \\
{[\mathrm{n}=55(22 \%)]}\end{array}$ & $\begin{array}{c}\text { Absent } \\
{[\mathrm{n}=192(78 \%)]}\end{array}$ & \\
\hline Age, years & $66.2(11.3)$ & $72.9(8.1)$ & $64.3(11.4)$ & $<0.001$ \\
\hline Men, n (\%) & $146(59 \%)$ & $37(67 \%)$ & $109(57 \%)$ & 0.16 \\
\hline Duration of dialysis, years & $7[3,15]$ & $5[3,11]$ & $7[3,16]$ & 0.04 \\
\hline Current smoker, n (\%) & $42(17 \%)$ & $11(20 \%)$ & $31(16 \%)$ & 0.50 \\
\hline Cause of end-stage kidney disease & & & & 0.45 \\
\hline Glomerulonephritis, n (\%) & $117(47 \%)$ & $22(40 \%)$ & $95(49 \%)$ & \\
\hline Diabetes, n (\%) & $61(25 \%)$ & $15(27 \%)$ & $46(24 \%)$ & \\
\hline Others, $\mathrm{n}(\%)$ & $69(28 \%)$ & $18(33 \%)$ & $51(27 \%)$ & \\
\hline \multicolumn{5}{|l|}{ Laboratory variables } \\
\hline Hemoglobin, g/dL & $10.4(1.2)$ & $10.4(1.2)$ & $10.5(1.2)$ & 0.69 \\
\hline Serum albumin, $g / d L$ & $3.8(0.5)$ & $3.6(0.4)$ & $3.8(0.5)$ & 0.02 \\
\hline Non-HDL cholesterol, mg/dL & $111(33)$ & $108(33)$ & $112(33)$ & 0.37 \\
\hline C-reactive protein, $\mathrm{mg} / \mathrm{dL}$ & $0.07[0.03,0.30]$ & $0.08[0.03,0.33]$ & $0.06[0.03,0.28]$ & 0.48 \\
\hline
\end{tabular}

Data are shown as number $(\%)$ for categorical variables and mean (standard deviation) or median [25th, 75th percentiles] for continuous variables, as appropriate.

HDL: high-density lipoprotein

time were used to estimate the associations between presence or absence of ELC and all-cause mortality, because the proportional hazards assumption was in doubt. The restricted mean survival time can be estimated simply by the area under the curve up to a given time $(18,19)$. All tests were two-tailed, with $\mathrm{p}<0.05$ considered statistically significant. All statistical analyses were performed using the software programs IBM SPSS Statistics for Windows (version 24.0; SPSS, Armonk, USA), Stata (version 15.1; Stata, College Station, USA), and R (version 3.2.5; R Project for Statistical Computing).

\section{Results}

A total of 247 hemodialysis patients without a history of CVD at baseline were included in this study. The participant selection process is detailed in Fig. 1. The mean (SD) patient age was 66.2 (11.3) years, 59\% were men, and the median dialysis vintage was 7 years (Table 1). The most common causes of ESKD were glomerulonephritis (47\%) and diabetes $(25 \%)$. Of the 247 patients, 55 (22\%) were identified as having ELC. Patients with ELC were more likely to be older, have shorter dialysis vintages, and exhibit lower serum albumin concentrations than those without ELC.

The mean follow-up time was 3.1 years for first cardiovascular events and 3.4 years for mortality. During the follow-up period, 43 patients suffered cardiovascular events [17.4\%; incidence rate of 5.6 per 100 person-years (95\% CI: 4.1-7.6)]. The cardiovascular events included myocardial infarction $(n=16)$, ischemic or hemorrhagic stroke $(n=21)$, and peripheral vascular disease requiring aortic or peripheral vascular bypass surgery or below- or above-the-knee amputa- 


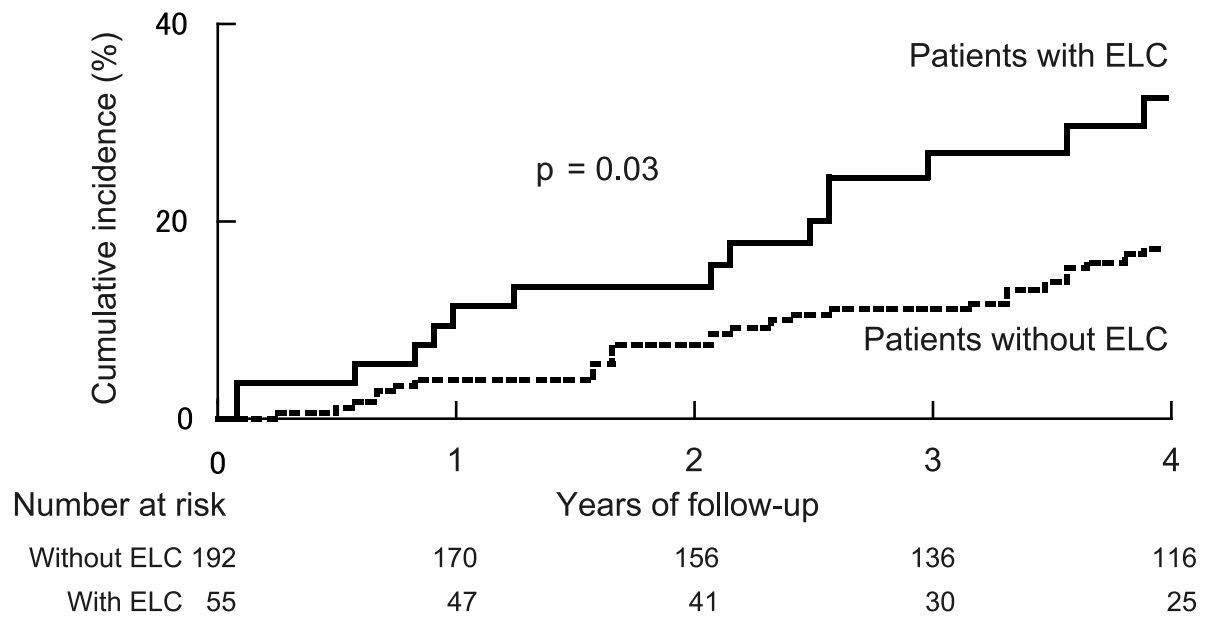

Figure 2. Cumulative incidence functions for cardiovascular events by the presence or absence of ELC (accounting for the competing risk of non-cardiovascular death), based on the competing risk regression model. Cardiovascular event: Fatal or nonfatal myocardial infarction, ischemic or hemorrhagic stroke, or peripheral vascular disease that required aortic or peripheral vascular bypass surgery or below- or above-the-knee amputation. Non-cardiovascular death: Death other than cardiovascular death or death from unknown causes (i.e., infection, malignancy, suicide, or unknown). ELC: earlobe crease

Table 2. Subhazard Ratios and 95\% Confidence Intervals (CIs) for Cardiovascular Events Using a Fine-Gray Proportional Subhazards Model.

\begin{tabular}{lcc}
\hline \multicolumn{1}{c}{ Model } & Subhazard ratio $(95 \% \mathrm{CI})$ & $\mathrm{p}$ value \\
\hline Unadjusted & $2.04(1.09-3.82)$ & 0.03 \\
Adjusted for age & $1.76(0.92-3.38)$ & 0.09 \\
Adjusted for age and sex & $1.67(0.83-3.38)$ & 0.15 \\
Adjusted for propensity-score & $1.56(0.78-3.11)$ & 0.21 \\
\hline
\end{tabular}

tion $(n=6)$. After the competing risk of non-cardiovascular death was taken into account, patients with ELC had an increased cumulative incidence of cardiovascular events compared to those without ELC (subhazard ratio $=2.04,95 \% \mathrm{CI}$ : 1.09-3.82) (Fig. 2). After adjusting for the age, however, the effect of ELC presence was no longer significant (Table 2). Propensity score-adjusted results also showed similar findings. When deaths due to unknown causes were classified as CVD deaths, similar results were observed (Supplementary material).

During the follow-up period, 47 patients died [19.0\%; mortality rate of 5.7 per 100 person-years (95\% CI: 4.27.5)], of whom 31 died without experiencing any cardiovascular events. The causes of death were CVD $(n=12)$, infection $(n=8)$, malignancy $(n=6)$, suicide $(n=1)$, others $(n=5)$, and unknown $(n=15)$. The Kaplan-Meier plot showed that patients with ELC had a higher crude all-cause mortality than those without ELC, and the proportional hazards assumption was in doubt (Fig. 3). A post-hoc analysis using a restricted mean survival time up to 48 months showed that the mean survival times (95\% CI) among patients with and without ELC were 41.3 months (38.0-44.6) and 44.4 months (42.9-45.9), respectively. No significant difference in the survival time up to 48 months was observed, with a difference of -3.1 months (95\% CI: -6.7 to 0.5 months; $p=0.09$ ).

\section{Discussion}

This prospective cohort study of Japanese hemodialysis patients without a history of CVD showed that patients with ELC had an increased risk of cardiovascular events, compared to those without ELC, although this difference was no longer significant after adjusting for age. These findings suggest that ELC has no independent association with cardiovascular events among Japanese hemodialysis patients and that these marks are simply indicative of advanced age. Furthermore, there were no significant survival differences between patients with and without ELC. Checking ELC may not be useful for identifying high-risk hemodialysis patients.

Previously, we reported that there was no significant association between the prevalence of ELC and CVD using cross-sectional data at baseline (15). In a cross-sectional study, however, survival bias can influence the findings. For this reason, we conducted a prospective cohort study and found no independent association between ELC and cardiovascular events. 


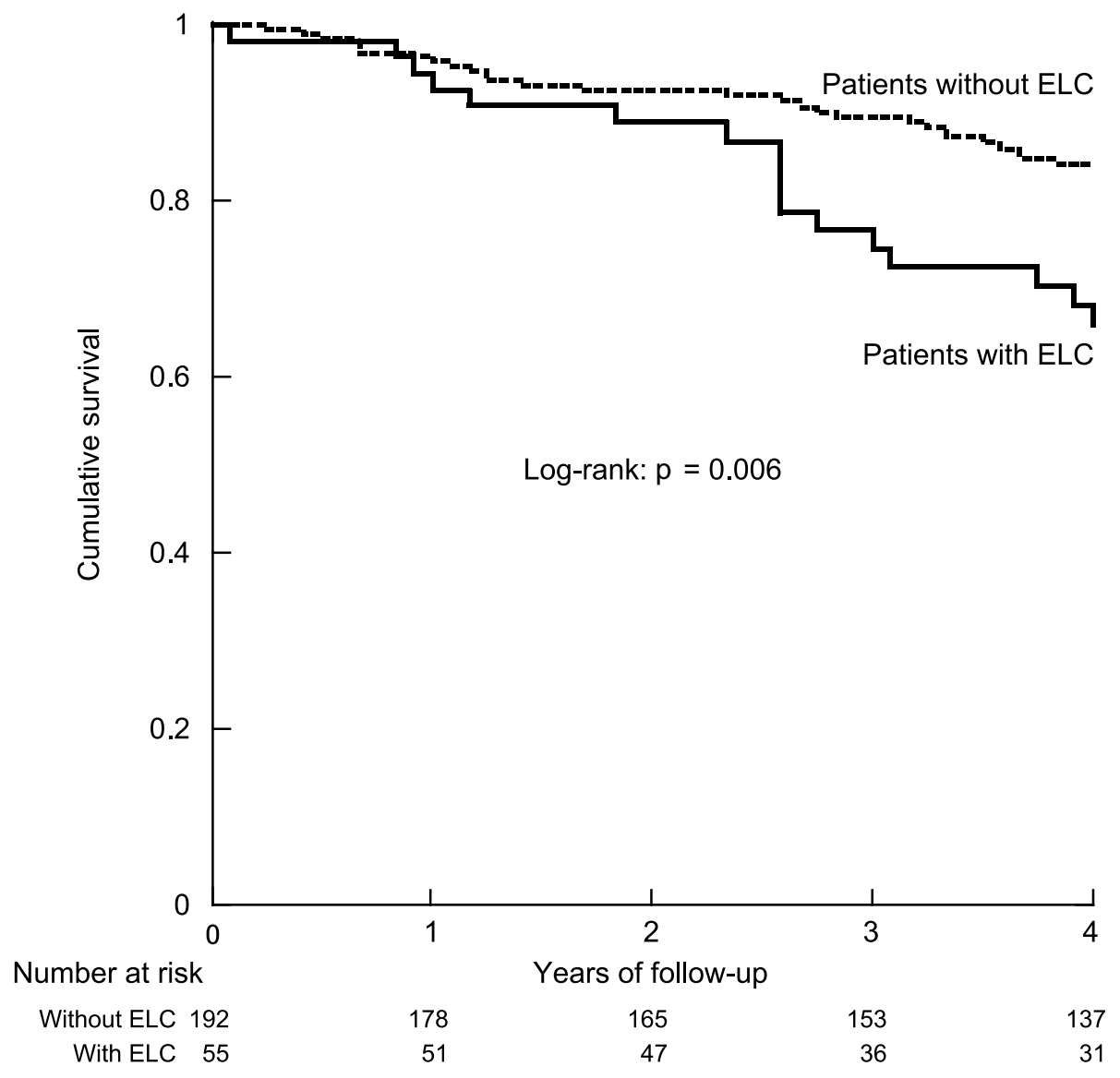

Figure 3. Cumulative survival by the presence or absence of ELC. ELC: earlobe crease

There are several possible explanations for the lack of an independent association between ELC and cardiovascular events in this study. First, ELC might simply be indicative of advanced age, as suggested by some studies among nondialysis populations. A prospective study of 261 consecutive men undergoing coronary arteriography showed no significant differences in the age-specific prevalence of coronary artery disease in men with and without ELC, suggesting that the reported association between ELC and coronary artery disease is because both ELC and coronary artery disease prevalence increase with age (8). A cross-sectional study of 863 community-dwelling adults $\geq 40$ years of age showed that the cognitive performance measured by the Montreal Cognitive Assessment (MoCA) did not differ markedly according to the presence or absence of ELC when stratified by age. This suggests that the association between ELC and cognitive performance is mainly related to age (20). A crosssectional study of 1,022 patients with type 2 diabetes showed that the presence of ELC was associated with increasing age, and that there was no independent association between the presence of ELC and the history of coronary artery disease after adjusting for cardiovascular risk factors, including age (21). These studies suggest that advancing age is responsible for this association, which may explain why the present study found no independent association between ELC and cardiovascular events.

Second, traditional cardiovascular risk factors (as opposed to non-traditional factors) may be closely associated with the development of ELC. This possibility would explain why the independent association between ELC and cardiovascular events was not significant in the present study. Although the mechanisms leading to the development of ELC are still unclear, previous studies in non-dialysis populations have shown ELC to be associated with well-known traditional cardiovascular risk factors, such as hypertension $(9,12-14)$, smoking $(9,13,22)$, obesity (22), and diabetes $(12,14,23)$. Hemodialysis patients have a higher prevalence of these traditional factors, as well as several non-traditional risk factors, such as anemia, hyperhomocysteinemia, chronic kidney disease-mineral bone disorder (CKD-MBD), oxidative stress, malnutrition, and chronic inflammation, than non-dialysis patients (24). The substantial differences in CVD risk factors between hemodialysis patients and non-dialysis populations may have affected the current findings.

This study has several limitations. First, information on cardiovascular events was collected from medical records. Although the majority of the records were complete, cardiovascular event rates may have been underestimated because asymptomatic events might have been missed. Second, this study included only Japanese patients without a history of CVD; therefore, the findings may not be generalizable to other dialysis populations. A cross-sectional study using police arrest photographs ("Mug shots") demonstrated that Chinese participants had a lower prevalence of ELC than 
white, black, or Latin-American participants (25). Furthermore, Japanese hemodialysis patients seldom have CVD, although a large proportion of patients undergoing maintenance hemodialysis in other countries may have CVD (24). A nationwide cohort study of Japanese dialysis patients showed that $7.2 \%, 4.5 \%$, and $14.4 \%$ of patients had a history of myocardial infarction, cerebral hemorrhaging, and cerebral infarction, respectively (26). An autopsy case report of a 60-year-old Japanese man who had received dialysis for 42 years indicated very little atheroma in his arterial tree, including the aorta and coronary arteries (27). These findings suggest that not all dialysis patients experience the burden of CVD in Japan. The association between ELC and cardiovascular events may differ among countries.

Despite these limitations, the current study has several strengths. First, the use of blinded evaluation methods in the assessment of ELC prevents observer bias. Second, to our knowledge, this is the first prospective study to assess the association between ELC and cardiovascular events among hemodialysis patients.

\section{Conclusion}

This study is the first to report that there is no independent association between ELC and cardiovascular events among Japanese hemodialysis patients, suggesting that advanced age may be responsible for the association between these factors.

The authors state that they have no Conflict of Interest (COI).

\section{Financial Support}

This study was supported in part by Grant-in-Aid for Scientific Research, KAKENHI (Grant Number JP23590781) received by MW from the Ministry of Education, Culture, Sports, Science and Technology of Japan.

\section{References}

1. Wakasugi M, Kazama JJ, Yamamoto S, et al. Cause-specific excess mortality among dialysis patients: comparison with the general population in Japan. Ther Apher Dial 17: 298-304, 2013.

2. Yoshino M, Kuhlmann MK, Kotanko P, et al. International differences in dialysis mortality reflect background general population atherosclerotic cardiovascular mortality. J Am Soc Nephrol 17: 3510-3519, 2006.

3. Roberts MA, Polkinghorne KR, McDonald SP, et al. Secular trends in cardiovascular mortality rates of patients receiving dialysis compared with the general population. Am J Kidney Dis $\mathbf{5 8}$ 64-72, 2011.

4. Pippias M, Jager KJ, Kramer A, et al. The changing trends and outcomes in renal replacement therapy: data from the ERA-EDTA Registry. Nephrol Dial Transplant 31: 831-841, 2016.

5. Wakasugi M, Kazama JJ, Narita I. Mortality trends among Japanese dialysis patients, 1988-2013: a joinpoint regression analysis. Nephrol Dial Transplant 31: 1501-1507, 2016.

6. Masakane I, Taniguchi M, Nakai S, et al. Annual dialysis data report 2015, JSDT renal data registry. Renal Replacement Therapy
4: $19,2018$.

7. Frank ST. Aural sign of coronary-artery disease. N Engl J Med 289: 327-328, 1973.

8. Brady PM, Zive MA, Goldberg RJ, et al. A new wrinkle to the earlobe crease. Arch Intern Med 147: 65-66, 1987.

9. Evrengul H, Dursunoglu D, Kaftan A, et al. Bilateral diagonal earlobe crease and coronary artery disease: a significant association. Dermatology 209: 271-275, 2004.

10. Christoffersen M, Frikke-Schmidt R, Schnohr P, et al. Visible agerelated signs and risk of ischemic heart disease in the general population: a prospective cohort study. Circulation 129: 990-998, 2014.

11. Wu XL, Yang DY, Zhao YS, et al. Diagonal earlobe crease and coronary artery disease in a Chinese population. BMC Cardiovasc Disord 14: 43, 2014.

12. Nazzal S, Hijazi B, Khalila L, et al. Diagonal earlobe crease (Frank's sign): a predictor of cerebral vascular events. Am J Med 130: 1324, 2017.

13. Ramos PM, Gumieiro JH, Miot HA. Association between ear creases and peripheral arterial disease. Clinics (Sao Paulo) 65: 1325-1327, 2010.

14. Rodriguez-Lopez C, Garlito-Diaz H, Madronero-Mariscal R, et al. Earlobe crease shapes and cardiovascular events. Am J Cardiol 116: $286-293,2015$

15. Wakasugi M, Kazama JJ, Kawamura K, et al. Prevalence of earlobe creases and their association with history of cardiovascular disease in patients undergoing hemodialysis: a cross-sectional study. Ther Apher Dial 21: 478-484, 2017.

16. Wakasugi $M$, Kawamura $K$, Yamamoto $S$, et al. High mortality rate of infectious diseases in dialysis patients: a comparison with the general population in Japan. Ther Apher Dial 16: 226-231, 2012.

17. Cepeda MS, Boston R, Farrar JT, et al. Comparison of logistic regression versus propensity score when the number of events is low and there are multiple confounders. Am J Epidemiol 158: 280287, 2003.

18. Zhao L, Claggett B, Tian L, et al. On the restricted mean survival time curve in survival analysis. Biometrics 72: 215-221, 2016.

19. Couchoud C, Dantony E, Elsensohn MH, et al. Restricted mean survival time over 15 years for patients starting renal replacement therapy. Nephrol Dial Transplant 32 (Suppl 2): ii60-ii67, 2017.

20. Del Brutto OH, Mera RM, Zambrano $M$, et al. The association between earlobe crease (Frank's sign) and cognitive performance is related to age. Results from the Atahualpa Project. Arch Gerontol Geriatr 79: 104-107, 2018.

21. Davis TM, Balme M, Jackson D, et al. The diagonal ear lobe crease (Frank's sign) is not associated with coronary artery disease or retinopathy in type 2 diabetes: the Fremantle Diabetes Study. Aust N Z J Med 30: 573-577, 2000.

22. Christoffersen M, Tybjaerg-Hansen A. Visible aging signs as risk markers for ischemic heart disease: epidemiology, pathogenesis and clinical implications. Ageing Res Rev 25: 24-41, 2016.

23. Kang EH, Kang HC. Association between earlobe crease and the metabolic syndrome in a cross-sectional study. Epidemiol Health 34: e2012004, 2012.

24. Cozzolino M, Mangano M, Stucchi A, et al. Cardiovascular disease in dialysis patients. Nephrol Dial Transplant 33 (Suppl 3): iii28-iii34, 2018.

25. Petrakis NL, Koo L. Earlobe crease. Lancet 1: 376, 1980.

26. Wakasugi M, Kazama JJ, Wada A, Hamano T, Masakane I, Narita I. Functional impairment attenuates the association between high serum phosphate and mortality in dialysis patients: a nationwide cohort study. Nephrol Dial Transplant 34: 1207-1216, 2019.

27. Suwabe $T$, Ubara $Y$, Inoue $M$, et al. What can we learn from a patient on dialysis for 42 years? Clin Nephrol 81: 427-434, 2014.

The Internal Medicine is an Open Access journal distributed under the Creative Commons Attribution-NonCommercial-NoDerivatives 4.0 International License. To view the details of this license, please visit (https://creativecommons.org/licenses/ by-nc-nd/4.0/).

(C) 2020 The Japanese Society of Internal Medicine

Intern Med 59: 927-932, 2020 